\title{
Effect of Strain Hardening Laws on Solution Behavior Near Frictional Interfaces in Metal Forming Processes: A Simple Analytical Example
}

\author{
Sergei Alexandrov ${ }^{1,2}$, Elena Lyamina ${ }^{1}$ and Pierre-Yves Manach ${ }^{3, *(1)}$ \\ 1 Ishlinsky Institute for Problems in Mechanics RAS, 119526 Moscow, Russia; \\ sergei_alexandrov@spartak.ru (S.A.); lyamina@inbox.ru (E.L.) \\ 2 Federal State Autonomous Educational Institution of Higher Education, South Ural State \\ University (National Research University), 454080 Chelyabinsk, Russia \\ 3 IRDL UMR CNRS 6027, Université Bretagne Sud, Rue de Saint Maudé, 56100 Lorient, France \\ * Correspondence: pierre-yves.manach@univ-ubs.fr
}

Received: 8 October 2020; Accepted: 15 November 2020; Published: 17 November 2020

\begin{abstract}
The main objective of the present paper is to compare, by means of a problem leading to a closed-form solution, the qualitative behavior of solutions based on three strain hardening laws: Swift's law, Ludwik's law, and Voce's law. The boundary value problem involves the maximum friction law as one of the boundary conditions. Such features of the solutions as nonexistence and singularity are emphasized. An important feature of Swift's and Ludwik's laws is that the equivalent stress approaches infinity as the equivalent strain approaches infinity. On the contrary, Voce's law involves saturation stress as one of the constitutive parameters. This qualitative difference in the equivalent stress behavior as the equivalent strain approaches infinity results in the qualitative difference in solutions' behavior. In particular, Swift's and Ludwik's hardening laws are compatible with the regime of sticking independently of other conditions. In the case of Voce's law, the solution under sticking conditions may break down. Moreover, Voce's law predicts intensive strain levels near the friction surface at sliding, and the other strain hardening laws do not. Thin layers of intensive plastic deformation often occur near frictional interfaces in metal forming processes. Voce's law predicts the occurrence of such layers without any additional assumptions.
\end{abstract}

Keywords: friction; singularity; work hardening; plasticity; metal forming processes

\section{Introduction}

Friction between tools and workpieces inevitably occurs in most metal forming processes and has a significant impact on these processes' performance, the quality of the product's surface, and other factors. Tresca's friction law is most often used as the friction boundary condition for modeling metal forming processes. This law postulates that the friction stress at sliding is equal to a portion of the local shear yield stress. A particular case of this friction law is the maximum friction law. In this case, the friction stress at sliding is equal to the local shear yield stress. The surface on which the maximum friction law applies is called the maximum friction surface. The qualitative behavior of solutions near the maximum friction surface is complex and includes nonexistence and singularity under certain conditions. These features of solutions may require special numerical methods for solving boundary value problems and the verification of assumptions that are usually used to simplify boundary value problems for solving by approximate analytical methods.

The qualitative behavior of solutions near maximum friction surfaces depends on the constitutive equations. Several boundary value problems that involve the maximum friction law have been formulated and solved analytically for rigid perfectly plastic models in [1,2]. The asymptotic analysis of 
these solutions shows that all of them are singular in the sense that the quadratic invariant of the strain rate tensor approaches infinity in the vicinity of the maximum friction surfaces. These solutions have been extended to the double-shearing model proposed in [3] in [4-6]. The qualitative behavior of these solutions is the same as that of the rigid perfectly plastic solutions. It has been shown in [7] that it is a general feature of rigid perfectly plastic solutions that the quadratic invariant of the strain rate tensor is inversely proportional to the square root of the distance to maximum friction surfaces. For this reason, such boundary value problems cannot be solved using traditional finite elements. The extended finite element method [8] should be capable of solving these problems. However, no attempt has yet been made. The strain rate intensity factor has been introduced in [7]. This factor controls the magnitude of the quadratic invariant of the strain rate tensor in the vicinity of maximum friction surfaces.

The solution behavior near maximum friction surfaces is more complicated in the case of rigid viscoplastic materials. For example, an analytic solution for compression of a viscoplastic layer between two parallel, rough plates has been proposed in [9]. This solution is based on the same assumptions as the solutions found in [1] and [4] for the rigid perfectly plastic and double-shearing models, respectively. In general, the solution given in [9] is a generalization of the solution in [1]. However, the solution in [9] does not exist if the maximum friction law applies. This is because the maximum friction law inevitably results in the regime of sticking at the maximum friction surface in the case of the constitutive equations adopted in [9]. A proof of this statement has been given in [10]. On the other hand, the assumptions accepted in [9] require the regime of sliding. This contradiction between the exact solution's qualitative behavior and the assumptions involved in the approximate solution leads to the nonexistence of the approximate solution. The regime of sliding at maximum friction surfaces is possible if the viscoplastic constitutive equations involve saturation stress [11,12]. Such solutions' qualitative behavior is affected by the dependence of the equivalent stress on the equivalent strain rate in the vicinity of the saturation stress. However, the quadratic invariant of the strain rate tensor approaches infinity in the vicinity of maximum friction surfaces in any case. The viscoplastic solution's asymptotic behavior may or may not coincide with that of the corresponding rigid perfectly plastic solution.

The asymptotic representation of solutions near maximum friction surfaces for other constitutive equations has been derived in $[13,14]$. All these solutions are singular.

The short review above shows the importance of understanding the exact asymptotic behavior of solutions near maximum friction surfaces. However, none of the results mentioned deal with work hardening constitutive equations. On the other hand, such material models are most often used to simulate cold metal forming processes. The present paper deals with a boundary value problem that can be considered as an approximation of the final stage of the hole-flanging process [15].

In addition to the fundamental aspects of continuum mechanics noted above, this research has practical applications. Thin, hardened layers of material are often generated in the vicinity of frictional interfaces in machining and deformation processes [16]. Standard tests used for determining material properties are not representative of the real material behavior within such layers [17]. In particular, shearing deformation is very intensive near frictional surfaces, whereas it is negligible in the uniaxial tension test that is usually used for determining the hardening law. A new approach for predicting the evolution of material properties in subsurface layers has been proposed in [18]. The approach is based on the strain rate intensity factor introduced in [7]. Several experimental studies have been carried out to apply this approach to specific materials [19-21]. However, there is an inconsistency between the experiments and theory. The experiments were carried out on strain hardening materials, whereas the theory is for rigid perfectly plastic material. The present paper attempts to smooth this inconsistency for one specific case. The theoretical solution derived can also be used in conjunction with the experimental results of the friction test proposed and developed in [22-24]. In these works and in [19-21], many micrographs illustrating the difference between the material microstructure near the friction surface and the bulk have been presented. 


\section{Statement of the Problem}

A tubular specimen of plastic material is confined between rigid solid and hollow cylinders (Figure 1). The inner and outer radii of the specimen are denoted as $a$ and $b$, respectively. The outer rigid cylinder moves with velocity $V$. The inner cylinder is motionless. The maximum friction law is assumed at the interfaces between each of the rigid cylinders and the specimen. End effects are neglected. Then, the deformation induced by the rigid cylinders is telescopic shearing. These assumptions are consistent with the modeling of the hole-flanging process with ironing conditions [15].

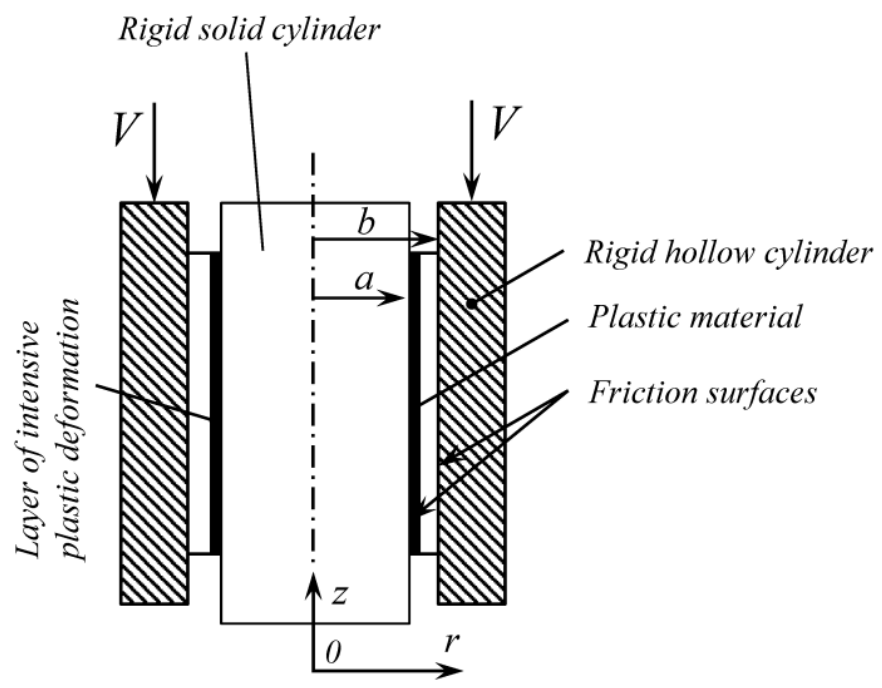

Figure 1. Schematic diagram of the process.

It is convenient to use a cylindrical coordinate system $(r, \theta, z)$, the $z$ axis of which coincides with the axis of symmetry of the process (Figure 1). In the case of telescopic shearing, the only nonzero strain rate component referred to this coordinate system is the shear strain rate $\xi_{r z}$.

Then, the strain rate tensor is

$$
\mathbf{E}=\left(\begin{array}{ccc}
0 & 0 & \xi_{r z} \\
0 & 0 & 0 \\
\xi_{r z} & 0 & 0
\end{array}\right) .
$$

Accordingly, the stress tensor is

$$
\boldsymbol{\Sigma}=\left(\begin{array}{ccc}
\sigma & 0 & \tau_{r z} \\
0 & \sigma & 0 \\
\tau_{r z} & 0 & \sigma
\end{array}\right) .
$$

Here, $\sigma$ is the hydrostatic stress and $\tau_{r z}$ is the only nonzero shear stress. The direction of the velocity vector $\mathbf{V}$ suggests that

$$
\tau_{r z}<0 \text { and } \xi_{r z}<0 .
$$

Without considering the anisotropy, which has little influence on this type of process, the solution is independent of both $\theta$ and $z$. Moreover, $\sigma$ is constant. Then, it follows from (2) that the only nontrivial equilibrium equation is

$$
\frac{\partial \tau_{r z}}{\partial r}+\frac{\tau_{r z}}{r}=0
$$

The equivalent stress, $\sigma_{e q}$, and the equivalent strain rate, $\xi_{e q}$, are defined as 


$$
\begin{gathered}
\sigma_{e q}=\sqrt{\frac{3}{2}} \sqrt{\left(\sigma_{r r}-\sigma\right)^{2}+\left(\sigma_{\theta \theta}-\sigma\right)^{2}+\left(\sigma_{z z}-\sigma\right)^{2}+2 \tau_{r \theta}^{2}+2 \tau_{z \theta}^{2}+2 \tau_{r z r}^{2}} \\
\xi_{e q}=\sqrt{\frac{2}{3}} \sqrt{\xi_{r r}^{2}+\xi_{\theta \theta}^{2}+\xi_{z z}^{2}+2 \xi_{r \theta}^{2}+2 \xi_{z \theta}^{2}+2 \xi_{r z}^{2}} .
\end{gathered}
$$

Using (1)-(3), Equation (5) transforms to

$$
\sigma_{e q}=-\sqrt{3} \tau_{r z} \quad \text { and } \quad \xi_{e q}=-\frac{2}{\sqrt{3}} \xi_{r z}
$$

The equivalent strain, $\varepsilon_{e q}$, is determined from the equation

$$
\frac{d \varepsilon_{e q}}{d t}=\xi_{e q}
$$

where $d / d t$ denotes the convected derivative. In the case of telescopic shearing, the only nonzero velocity component is the axial velocity $u_{z}=u$. Therefore,

$$
\xi_{r z}=\frac{1}{2} \frac{\partial u}{\partial r}
$$

Using (8) and the second equation in (6), one can rewrite (7):

$$
\frac{d \varepsilon_{e q}}{d t}=-\frac{1}{\sqrt{3}} \frac{\partial u}{\partial r}
$$

The constitutive equations of rigid plastic, strain hardening material comprise a yield criterion and its associated flow rule. Equation (2) implies that any yield criterion for incompressible material reduces to

$$
\sigma_{e q}=\sigma_{0} \Phi\left(\varepsilon_{e q}\right)
$$

where $\sigma_{0}$ is the initial yield stress in tension and $\Phi\left(\varepsilon_{e q}\right)$ is an arbitrary function of its argument satisfying the conditions $\Phi=1$ at $\varepsilon_{e q}=0$ and $d \Phi\left(\varepsilon_{e q}\right) / d \varepsilon_{e q} \geq 0$ for all $\varepsilon_{e q}$. The associated flow rule is automatically satisfied due to (1)-(3).

The equivalent strain vanishes on rigid/plastic boundaries. Therefore, it is seen from (10) that

$$
\sigma_{e q}=\sigma_{0}
$$

on such boundaries.

\section{General Solution}

The maximum friction law allows two regimes: sticking and sliding. One should find from the solution which regime occurs on each friction surface. Equations (3) and (4) show that $\partial \tau_{r z} / \partial r>0$ everywhere. Therefore, $\left|\tau_{r z}\right|$ attains its maximum value at $r=a$. Then, there are two possible cases: (i) the regime of sticking occurs on both friction surfaces, and (ii) the regime of sticking occurs at $r=b$ and the regime of sliding at $r=a$. In either case, one of the velocity boundary conditions is

$$
u=-V
$$

for $r=b$. The specific form of the boundary condition at $r=a$ depends on the regime of friction. In Case (i),

$$
u=0
$$


for $r=a$. If there is a rigid region, then the boundary condition (12) transforms to

$$
u=-V
$$

for $r=r_{c}$. Here, $r_{c}$ is the radius of the rigid/plastic boundary. In Case (ii), the boundary condition at $r=a$ is formulated in terms of stresses and depends on the constitutive equations.

Equations (4) and (6) combine to give

$$
\frac{\partial \sigma_{e q}}{\partial r}+\frac{\sigma_{e q}}{r}=0
$$

Since the velocity vector is parallel to the $z$ axes and the solution is independent of $z$, $d \varepsilon_{e q} / d t=\partial \varepsilon_{e q} / \partial t$. This equation, together with (9), leads to

$$
\frac{\partial \varepsilon_{e q}}{\partial t}=-\frac{1}{\sqrt{3}} \frac{\partial u}{\partial r} .
$$

At the beginning of the process, a plastic region initiates at $r=a$ and then propagates to the surface $r=b$. This stage of the process lasts until the rigid/plastic boundary reaches the surface $r=b$. The solution of Equation (15) satisfying the condition (11) is

$$
\frac{\sigma_{e q}}{\sigma_{0}}=\frac{r_{c}}{r}
$$

Substituting (10) into (17) yields

$$
\Phi\left(\varepsilon_{e q}\right)=\frac{r_{c}}{r} \quad \text { or } \quad \varepsilon_{e q}=\Lambda\left(\frac{r_{c}}{r}\right)
$$

where $\Lambda$ is the function inverse to $\Phi$.

The solution above is written in terms of the independent variables $t$ and $r$. It is convenient to continue to solve the boundary value problem for the stage of the process under consideration in terms of the following independent variables:

$$
\rho=\frac{r_{c}}{r} \quad \text { and } \quad s .
$$

Here, $s$ is the distance traveled by the outer cylinder from its initial position. It is evident that $d s / d t=V$. Then, using (19), one can find

$$
\frac{\partial}{\partial t}=V\left(\frac{\partial}{\partial s}+\frac{\rho}{r_{c}} \frac{d r_{c}}{d s} \frac{\partial}{\partial \rho}\right) \text { and } \frac{\partial}{\partial r}=-\frac{\rho^{2}}{r_{c}} \frac{\partial}{\partial \rho} .
$$

The second equation in (18) becomes $\varepsilon_{e q}=\Lambda(\rho)$. Thus, the equivalent strain is independent of $s$ in the new independent variables. Then, Equations (16) and (20) combine to give

$$
\frac{\partial u}{\partial \rho}=\frac{\sqrt{3} V}{\rho} \frac{d r_{c}}{d s} \frac{\partial \varepsilon_{e q}}{\partial \rho}
$$

Eliminating the equivalent strain in this equation by means of the second equation in (18) results in

$$
\frac{\partial u}{\partial \rho}=\frac{\sqrt{3} V}{\rho} \frac{d r_{c}}{d s} \frac{d \Lambda}{d \rho}
$$


In the new independent variables, the boundary condition (14) becomes $u=-V$ for $\rho=1$. The solution of Equation (22) satisfying this boundary condition is

$$
\frac{u}{V}=\sqrt{3} \frac{d r_{c}}{d s} \int_{1}^{\rho} \frac{d \Lambda}{\omega d \omega} d \omega-1 .
$$

Here, $\omega$ is a dummy variable of integration. In the new independent variables, the boundary condition (13) becomes $u=0$ for $\rho=r_{c} / a$. The solution (23) and this boundary condition combine to give

$$
\frac{d r_{c}}{d s} \int_{1}^{r_{c} / a} \frac{d \Lambda}{\rho d \rho} d \rho=\frac{1}{\sqrt{3}} .
$$

Integrating by parts and taking into account that $\Lambda=0$ at $\rho=1$, one can find

$$
\frac{d s}{d r_{c}}=\sqrt{3}\left[\int_{1}^{r_{c} / a} \frac{\Lambda(\rho)}{\rho^{2}} d \rho+\frac{a}{r_{c}} \Lambda\left(\frac{r_{c}}{a}\right)\right] .
$$

This is an ordinary differential equation for finding $s$ as a function of $r_{c}$. The initial condition to this equation is

$$
s=0
$$

at $r_{c}=a$. The solution of Equation (25) satisfying this initial condition is

$$
\frac{s}{\sqrt{3}}=r_{c} \int_{1}^{r_{c} / a} \frac{\Lambda(\rho)}{\rho^{2}} d \rho .
$$

The solution above is valid if $r_{c} \leq b$. Therefore, this stage of the process ends when $r_{c}=b$. The corresponding value of $s$ is determined from (27) as

$$
\frac{s_{1}}{\sqrt{3}}=b \int_{1}^{b / a} \frac{\Lambda(\rho)}{\rho^{2}} d \rho
$$

It is also seen from (17) that

$$
\frac{\sigma_{e q}}{\sigma_{0}}=\frac{b}{a}
$$

on the surface $r=a$ at this instant.

If $s \geq s_{1}$, then the plastic region occupies the domain $a \leq r \leq b$. The independent variables introduced in (19) cannot be used for solving the boundary value problem during this stage of the process. The solution of Equation (15) can be written as

$$
\frac{\sigma_{e q}}{\sigma_{0}}=\frac{a \alpha}{r}
$$

where $\alpha \sigma_{0}$ is the value of $\sigma_{e q}$ at $r=a$. The value of $\alpha$ should be found from the solution. Substituting (30) into (10) yields

$$
\Phi\left(\varepsilon_{e q}\right)=\frac{\alpha a}{r} \quad \text { or } \quad \varepsilon_{e q}=\Lambda\left(\frac{\alpha a}{r}\right)
$$


Introduce the following independent variables:

$$
\gamma=\frac{\alpha a}{r} \text { and } s
$$

Then,

$$
\frac{\partial}{\partial t}=V\left(\frac{\partial}{\partial s}+\frac{\gamma}{\alpha} \frac{d \alpha}{d s} \frac{\partial}{\partial \gamma}\right) \text { and } \frac{\partial}{\partial r}=-\frac{\gamma^{2}}{a \alpha} \frac{\partial}{\partial \gamma}
$$

The second equation in (31) becomes $\varepsilon_{e q}=\Lambda(\gamma)$. Thus, the equivalent strain is independent of $s$ in the new independent variables. Then, Equations (16) and (20) combine to give

$$
V \frac{d \alpha}{d s} \frac{\partial \varepsilon_{e q}}{\partial \gamma}=\frac{\gamma}{\sqrt{3} a} \frac{\partial u}{\partial \gamma}
$$

Eliminating the equivalent strain in this equation by means of the second equation in (31) results in

$$
\frac{\gamma}{\sqrt{3} a} \frac{\partial u}{\partial \gamma}=V \frac{d \alpha}{d s} \frac{d \Lambda}{d \gamma}
$$

In the new independent variables, the boundary condition (12) becomes $u=-V$ for $\gamma=\gamma_{a}=a \alpha / b$. The solution of Equation (35) satisfying this boundary condition is

$$
\frac{u}{V}=\sqrt{3} a \frac{d \alpha}{d s} \int_{\gamma_{a}}^{\gamma} \frac{d \Lambda}{\omega d \omega} d \omega-1
$$

In the new independent variables, the boundary condition (13) becomes $u=0$ for $\gamma=\alpha$. The solution (36) and this boundary condition combine to give

$$
\frac{d s}{d \alpha}=\sqrt{3} a \int_{\gamma a}^{\alpha} \frac{d \Lambda}{\gamma d \gamma} d \gamma
$$

Integrating by parts,

$$
\frac{d s}{d \alpha}=\sqrt{3} a\left[\int_{\gamma_{a}}^{\alpha} \frac{\Lambda(\gamma)}{\gamma^{2}} d \gamma+\frac{\Lambda(\alpha)}{\alpha}-\frac{\Lambda\left(\gamma_{a}\right)}{\gamma_{a}}\right]
$$

This is an ordinary differential equation for finding $s$ as a function of $\alpha$. The initial condition to this equation is determined from (29) and (30) as

$$
s=s_{1}
$$

at $\alpha=b / a$. The solution of Equation (38) satisfying this initial condition is

$$
s=\sqrt{3} a\left[\alpha \int_{\gamma_{a}}^{\alpha} \frac{\Lambda(\gamma)}{\gamma^{2}} d \gamma-\frac{b}{a} \int_{1}^{b / a} \frac{\Lambda(\gamma)}{\gamma^{2}} d \gamma\right]+s_{1}
$$

If $s$ is given, then Equation (40) serves for determining the corresponding value of $\alpha$. This equation may or may not have a solution. If the solution exists, then the regime of sticking always occurs at $r=a$ (i.e., Case (ii) never occurs). If the solution breaks down at a certain value of $s=s_{2}$, then one 
should search for a solution for Case (ii) in the range $s>s_{2}$. The precise structure of the solution depends on the function $\Lambda$.

\section{Effect of Strain Hardening Laws on Solution Behavior}

It will be seen later that the qualitative behavior of the solution near the friction surface is controlled by the qualitative behavior of the function $\Phi\left(\varepsilon_{e q}\right)$ involved in (10) as $\varepsilon_{e q} \rightarrow \infty$. In what follows, two types of this function will be considered (Figure 2). Curve 1 represents the hardening laws that predict that the yield stress increases with no limit as $\varepsilon_{e q} \rightarrow \infty$. Curve 2 represents the type of hardening laws that involve the saturation stress (i.e., the yield stress approaches the saturation stress as $\left.\varepsilon_{e q} \rightarrow \infty\right)$.

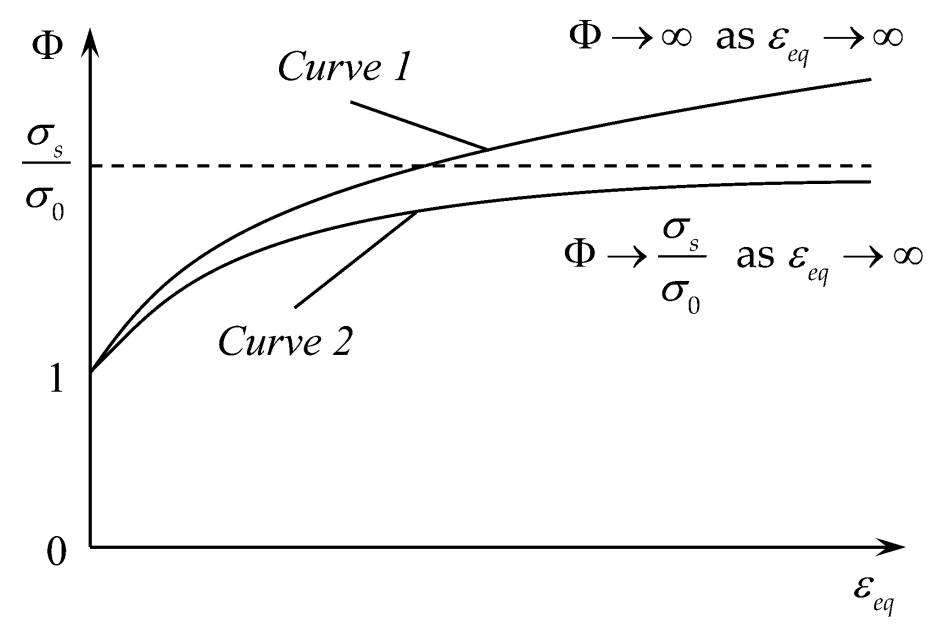

Figure 2. Geometric representation of qualitatively different hardening laws considered in the present paper.

\subsection{Swift's Law}

In this case, the function $\Phi\left(\varepsilon_{e q}\right)$ involved in (10) is

$$
\Phi\left(\varepsilon_{e q}\right)=\left(1+\frac{\varepsilon_{e q}}{\varepsilon_{0}}\right)^{n}
$$

where $\varepsilon_{0}>0$ and $n>0$ are constitutive parameters. Then, the function $\Lambda(\gamma)$ involved in (40) is

$$
\Lambda(\gamma)=\left(\gamma^{1 / n}-1\right) \varepsilon_{0} .
$$

Eliminating $\Lambda(\gamma)$ in (40) using (42) yields

$$
s=\frac{\sqrt{3} a n \varepsilon_{0}}{(1-n)}\left\{\alpha^{1 / n}\left[1-\left(\frac{a}{b}\right)^{n_{1}}\right]+\frac{b}{a}\left[1-\left(\frac{b}{a}\right)^{n_{1}}\right]\right\}+s_{1}
$$

where $n_{1}=(1-n) / n$. Equation (43) is valid in the range $\alpha \geq b / a$. It is straightforward to express $\alpha$ as a function of $s$ from (43). However, it is evident from (43) that this equation has a solution for $\alpha$ at any $s$ satisfying the inequality $s_{1} \leq s<\infty$. Therefore, the solution of the original boundary value problem satisfying the regime of sticking at $r=a$ always exists.

\subsection{Ludwik's Law}

In this case, the functions $\Phi\left(\varepsilon_{e q}\right)$ and $\Lambda(\gamma)$ are 


$$
\Phi\left(\varepsilon_{e q}\right)=1+\left(\frac{\varepsilon_{e q}}{\varepsilon_{0}}\right)^{n} \text { and } \Lambda(\gamma)=\varepsilon_{0}(\gamma-1)^{1 / n}
$$

Eliminating $\Lambda(\gamma)$ in (40) using (44) yields

$$
s=\sqrt{3} a \varepsilon_{0}\left[\alpha \int_{\gamma_{a}}^{\alpha} \frac{(\gamma-1)^{1 / n}}{\gamma^{2}} d \gamma-\frac{b}{a} \int_{1}^{b / a} \frac{(\gamma-1)^{1 / n}}{\gamma^{2}} d \gamma\right]+s_{1}
$$

It is seen from (37) that $d s / d \alpha>0$ for all $\alpha$. Therefore, the right-hand side of (45) is a monotonically increasing function of $\alpha$. The second term in the brackets in (45) is finite. Consider the integral involved in the first term in the brackets. Using the definition for $\gamma_{a}$, one can get

$$
\lim _{\alpha \rightarrow \infty} \int_{\alpha a / b}^{\alpha} \frac{(\gamma-1)^{1 / n}}{\gamma^{2}} d \gamma=\lim _{\alpha \rightarrow \infty} \int_{\alpha a / b}^{\alpha} \gamma^{\left(\frac{1}{n}-2\right)} d \gamma=\frac{n}{(1-n)}\left[1-\left(\frac{a}{b}\right)^{\left(\frac{1}{n}-1\right)}\right] \lim _{\alpha \rightarrow \infty}\left[\alpha^{\left(\frac{1}{n}-1\right)}\right] .
$$

Since $a<b$,

$$
\frac{n}{(1-n)}\left[1-\left(\frac{a}{b}\right)^{\left(\frac{1}{n}-1\right)}\right]=A>0
$$

if $n \neq 1$. Equations (46) and (47) combine to give

$$
\lim _{\alpha \rightarrow \infty}\left[\alpha \int_{\alpha a / b}^{\alpha} \frac{(\gamma-1)^{1 / n}}{\gamma^{2}} d \gamma\right]=A \lim _{\alpha \rightarrow \infty} \alpha^{1 / n} \rightarrow \infty
$$

if $n \neq 1$. Comparing (45) and (48) shows that

$$
s \rightarrow \infty
$$

as $\alpha \rightarrow \infty$ if $n \neq 1$. If $n=1$, then (45) becomes

$$
s=\sqrt{3} a \varepsilon_{0}\left[\left(1-\frac{a}{b}\right) \alpha \ln \alpha-\frac{b}{a} \ln \left(\frac{b}{a}\right)\right]+s_{1} .
$$

Thus, (49) is valid for any $n$ in the range $n>0$. Therefore, the solution of the original boundary value problem satisfying the regime of sticking at $r=a$ always exists.

\subsection{Voce's Law}

In this case, the functions $\Phi\left(\varepsilon_{e q}\right)$ and $\Lambda(\gamma)$ are

$$
\Phi\left(\varepsilon_{e q}\right)=\frac{\sigma_{s}}{\sigma_{0}}-\left(\frac{\sigma_{s}}{\sigma_{0}}-1\right) \exp \left(-\frac{\varepsilon_{e q}}{\varepsilon_{0}}\right) \text { and } \Lambda(\gamma)=\varepsilon_{0} \ln \left(\frac{\sigma_{s} / \sigma_{0}-1}{\sigma_{s} / \sigma_{0}-\gamma}\right) .
$$

Here, $\sigma_{s}$ is the saturation stress. In this case, it is more convenient to deal with Equation (37). Substituting (51) into this equation and integrating, one gets

$$
\frac{d s}{d \alpha}=-\frac{\sqrt{3} a \varepsilon_{0}}{c} \ln \left(\frac{c-\alpha}{c / m-\alpha}\right)
$$

where $c=\sigma_{s} / \sigma_{0}$ and $m=a / b$. The solution of Equation (52) satisfying the initial condition (39) is 


$$
\begin{aligned}
& s=-\frac{\sqrt{3} a \varepsilon_{0}}{c}[\left.(\alpha-c) \ln (c-\alpha)+\left(\frac{c}{m}-\alpha\right) \ln \left(\frac{c}{m}-\alpha\right)\right]+ \\
& \frac{\sqrt{3} a \varepsilon_{0}}{c}\left[\left(\frac{1}{m}-c\right) \ln \left(c-\frac{1}{m}\right)+\left(\frac{c-1}{m}\right) \ln \left(\frac{c-1}{m}\right)\right]+s_{1} .
\end{aligned}
$$

This solution is valid if

$$
c>1 / m \text { or } \frac{\sigma_{s}}{\sigma_{0}}>\frac{b}{a} .
$$

Since $m<1$, it is seen from (53) that the solution breaks down and the regime of sliding occurs at

$$
\alpha=c=\sigma_{s} / \sigma_{0} .
$$

Then, the maximum possible value of $s$ is determined from (53) as

$$
s_{\max }=-\sqrt{3} a \varepsilon_{0}\left[\left(\frac{1}{m}-1\right) \ln \left(\frac{c}{m}-c\right)\right]+\frac{\sqrt{3} a \varepsilon_{0}}{c}\left[\left(\frac{1}{m}-c\right) \ln \left(c-\frac{1}{m}\right)+\left(\frac{c-1}{m}\right) \ln \left(\frac{c-1}{m}\right)\right]+s_{1} .
$$

If $s>s_{\max }$, then the regime of sliding occurs at the surface $r=a$. The distribution of stresses and velocities is independent of $s$ in the range $s \geq s_{\max }$.

If the inequality (54) is not satisfied, then the regime of sliding at the surface $r=a$ occurs when $r_{c} \leq b$. Substituting (51) into (24) and integrating yields

$$
\frac{d s}{d R_{c}}=\frac{\sqrt{3} a \varepsilon_{0}}{c} \ln \left[\frac{(c-1) R_{c}}{c-R_{c}}\right]
$$

where $R_{c}=r_{c} / a$. The solution of Equation (57) satisfying the initial condition (26) is

$$
s=\frac{\sqrt{3} a \varepsilon_{0}}{c}\left\{R_{c} \ln \left[R_{c}(c-1)\right]+\left(c-R_{c}\right) \ln \left(c-R_{c}\right)-c \ln (c-1)\right\}
$$

It is seen from this equation that the solution breaks down and the regime of sliding occurs at

$$
R_{c}=c \quad \text { or } \quad r_{c}=a \sigma_{s} / \sigma_{0}
$$

Therefore, this solution (58) is valid if $\sigma_{s} / \sigma_{0} \leq b / a$, which is in agreement with (54). The maximum possible value of $s$ that follows from (58) using the definition for $c$ is

$$
s_{\max }=\sqrt{3} a \varepsilon_{0} \ln \left(\frac{\sigma_{s}}{\sigma_{0}}\right) .
$$

Equation (56) reduces to (60) at $m=1 / c$ or $a / b=\sigma_{0} / \sigma_{s}$.

The distribution of the equivalent strain is singular near the friction surface at sliding. It follows from (18) and (51) that

$$
\varepsilon_{e q}=\varepsilon_{0} \ln \left(\frac{\sigma_{s} / \sigma_{0}-1}{\sigma_{s} / \sigma_{0}-r_{c} / r}\right)
$$

if the solution (58) is valid. In the case of sliding, Equation (61) transforms using (59) to

$$
\varepsilon_{e q}=\varepsilon_{0} \ln \left[\frac{\sigma_{s} / \sigma_{0}-1}{\sigma_{s} / \sigma_{0}(1-a / r)}\right]=O|\ln (r-a)|
$$

as $r \rightarrow a$. The same result follows from (31), (51), and (55). Thus, the equivalent strain rate approaches infinity in the vicinity of the maximum friction surface at sliding. 


\subsection{Rigid Perfectly Plastic Material}

In the case of rigid perfectly plastic material, $\Phi\left(\varepsilon_{e q}\right)=1$. Therefore, Equation (15) is not satisfied in plastic regions of finite size. Therefore, the only possible solution is that the material is rigid in the region $a<r \leq b$ and $r=a$ is a velocity discontinuity surface. It follows from the general theory of plasticity that $\left|\tau_{r z}\right|=\sigma_{0} / \sqrt{3}$ on this surface [1].

The model of rigid plastic, hardening material based on the hardening laws in (41) and (44) reduces to the model of rigid perfectly plastic material if $1 / \varepsilon_{0}=0$. However, the corresponding solutions do not reduce to the rigid perfectly plastic solution, independently of how close the parameter $1 / \varepsilon_{0}=0$ is to zero. This conclusion is immediate from the solutions given in Sections 4.1 and 4.2.

The model of rigid plastic, hardening material based on the hardening law in (51) reduces to the model of rigid perfectly plastic material if $\sigma_{0}=\sigma_{s}$. In this case, it follows from (60) that $s_{\max }=0$, which is in agreement with the rigid perfectly plastic solution.

It is worthy of note that the same scenario occurs when the rigid perfectly plastic solution compares to viscoplastic solutions [25]. The solution for viscoplastic models with no saturation stress does not reduce to the rigid perfectly plastic solution. The solution for a class of viscoplastic models with saturation stress may reduce to the rigid perfectly plastic solution.

\section{Conclusions}

The initial/boundary value problem for the flow of the material resulting from the problem formulated in Section 2 has been solved in Sections 3 and 4, with the resulting solution being in closed form. The general solution given in Section 3 is valid for any strain hardening law. However, without specifying the strain hardening law, it is impossible to determine which regime of friction-sticking or sliding-occurs at the tubular specimen's inner surface.

Three widely used hardening laws have been considered in Section 4: Swift's law, Ludwik's law, and Voce's law. The first two predict that the equivalent stress approaches infinity as the equivalent strain approaches infinity. The third one involves saturation stress. This qualitative difference in the equivalent stress behavior as the equivalent strain approaches infinity results in the qualitative difference in solutions' behavior. In particular, Equations (43) and (49) show that Swift's and Ludwik's laws are always compatible with the regime of sticking at the maximum friction surface. On the other hand, the analysis of the general solution in Section 4.3 demonstrates that the solution based on Voce's law requires sliding under certain conditions. It is worthy of note that this requirement is a feature of the constitutive equation rather than the friction law.

In the case of Voce's law, the solution at sliding is singular. In particular, the equivalent strain approaches infinity in the vicinity of the maximum friction surface, and Equation (62) shows the exact asymptotic expansion of the equivalent strain near this surface. This feature of the solution is in qualitative agreement with experimental results on the generation of hardened layers in the vicinity of frictional interfaces in deformation processes [16]. The solutions for Swift's and Ludwik's laws do not have such a feature. Therefore, it is reasonable to hypothesize that Voce's law and, probably, other strain hardening laws that involve saturation stress are capable of predicting the generation of hardened layers near frictional interfaces without any additional assumptions, whereas the strain hardening laws with no saturation stress are not.

The effect of temperature has not been considered in the present paper. However, the temperature of the metal rises during plastic deformation because of the heat generated by mechanical work. It is seen from (62) that the temperature gradient should be quite large in the friction surface's vicinity. This feature of the temperature field has already been demonstrated for rigid/perfectly plastic and viscoplastic solids [26,27]. For this reason, the material can soften in the vicinity of frictional interfaces and hardening laws represented by curve 1 in Figure 2 can approach the hardening laws represented by curve 2 . 
Author Contributions: Conceptualization, P.-Y.M.; writing, S.A.; formal analysis, E.L. All authors have read and agreed to the published version of the manuscript.

Funding: This research was made possible by the grant RSF-18-19-00736.

Conflicts of Interest: The authors declare no conflict of interest.

\section{References}

1. Hill, R. The Mathematical Theory of Plasticity; Oxford University Press: New York, NY, USA, 1950.

2. Shield, R.T. Plastic flow in a converging conical channel. J. Mech. Phys. Solids 1955, 3, 246-258. [CrossRef]

3. Spencer, A.J.M. A theory of the kinematics of ideal soils under plane strain conditions. J. Mech. Phys. Solids 1964, 12, 337-351. [CrossRef]

4. Pemberton, C.S. Flow of imponderable granular materials in wedge-shaped channels. J. Mech. Phys. Solids 1965, 13, 351-360. [CrossRef]

5. Marshall, E.A. The compression of a slab of ideal soil between rough plates. Acta Mech. 1967, 3, 82-92. [CrossRef]

6. Spencer, A.J.M. Deformation of Ideal Granular Materials. In Mechanics of Solids, The Rodney Hill 60th Anniversary Volume; Hopkins, H.G., Sewell, M.J., Eds.; Pergamon Press: Oxford, UK, 1982; pp. 607-652.

7. Alexandrov, S.; Richmond, O. Singular plastic flow fields near surfaces of maximum friction stress. Int. J. Non Linear Mech. 2001, 36, 1-11. [CrossRef]

8. Fries, T.-P.; Belytschko, T. The extended/generalized finite element method: An overview of the method and its applications. Int. J. Numer. Methods Eng. 2010, 84, 253-304. [CrossRef]

9. Adams, M.J.; Briscoe, B.J.; Corfield, G.M.; Lawrence, C.J.; Papathanasiou, T.D. An analysis of the plane-strain compression of viscous materials. Trans. ASME J. Appl. Mech. 1997, 64, 420-424. [CrossRef]

10. Alexandrov, S.; Alexandrova, N. On the maximum friction law in viscoplasticity. Mech. Time Depend. Mater. 2000, 4, 99-104. [CrossRef]

11. Alexandrov, S.; Mishuris, G. Viscoplasticity with a saturation stress: Distinguished features of the model. Arch. Appl. Mech. 2007, 77, 35-47. [CrossRef]

12. Alexandrov, S.; Mishuris, G. Qualitative behaviour of viscoplastic solutions in the vicinity of maximum-friction surfaces. J. Eng. Math. 2009, 65, 143-156. [CrossRef]

13. Alexandrov, S.; Jeng, Y.-R. Singular rigid/plastic solutions in anisotropic plasticity under plane strain conditions. Cont. Mech. Therm. 2013, 25, 685-689. [CrossRef]

14. Wang, Y.; Alexandrov, S.; Lyamina, E. Solution behavior in the vicinity of characteristic envelopes for the double slip and rotation model. Appl. Sci. 2020, 10, 3220. [CrossRef]

15. Kacem, A.; Krichen, A.; Manach, P.Y. Occurrence and effect of ironing in the hole-flanging process. J. Mater. Process Technol. 2011, 211, 1606-1613. [CrossRef]

16. Griffiths, B.J. Mechanisms of white layer generation with reference to machining and deformation processes. Trans. ASME J. Tribol. 1987, 109, 525-530. [CrossRef]

17. Jaspers, S.P.F.C.; Dautzenberg, J. Material behaviour in conditions similar to metal cutting: Flow stress in the primary shear zone. Mater. Process Technol. 2002, 122, 322-330. [CrossRef]

18. Goldstein, R.V.; Alexandrov, S.E. An approach to prediction of microstructure formation near friction surfaces at large plastic strains. Phys. Mesomech. 2015, 18, 223-227. [CrossRef]

19. Alexandrov, S.; Jeng, Y.-R.; Hwang, Y.-M. Generation of a fine grain layer in the vicinity of frictional interfaces in direct extrusion of AZ31 alloy. ASME J. Manuf. Sci. Eng. 2015, 137. [CrossRef]

20. Alexandrov, S.; Sidjanin, L.; Vilotic, D.; Movrin, D.; Lang, L. Generation of a layer of severe plastic deformation near friction surfaces in upsetting of steel specimens. Metals 2018, 8, 71. [CrossRef]

21. Stolyarov, A.; Polyakova, M.; Atangulova, G.; Alexandrov, S.; Lang, L. Effect of frictional conditions on the generation of fine grain layers in drawing of thin steel wires. Metals 2019, 9, 819. [CrossRef]

22. Sanabria, V.; Mueller, S.; Reimers, W. A new high speed friction test for extrusion processes. Key Eng. Mater. 2014, 585, 33-39. [CrossRef]

23. Sanabria, V.; Mueller, S.; Reimers, W. Microstructure evolution of friction boundary layer during extrusion of AA 6060. Procedia Eng. 2014, 81, 586-591. [CrossRef]

24. Sanabria, V.; Gensch, F.; Mueller, S. Application of friction shear test for constitutive modeling evaluation of magnesium alloy AZ31B at high temperature. Procedia Manuf. 2020, 47, 237-244. [CrossRef] 
25. Alexandrov, S.; Miszuris, W. The transition of qualitative behaviour between rigid perfectly plastic and viscoplastic solutions. J. Eng. Math. 2016, 97, 67-81. [CrossRef]

26. Alexandrov, S.; Miszuris, W. Heat generation in plane strain compression of a thin rigid plastic layer. Acta Mech. 2016, 227, 813-821. [CrossRef]

27. Alexandrov, S.; Miszuris, W.; Lang, L. An efficient method of analysis of heat transfer during plane strain upsetting of a viscoplastic strip. ZAMM 2019, 99, e201700313. [CrossRef]

Publisher's Note: MDPI stays neutral with regard to jurisdictional claims in published maps and institutional affiliations.

(C) 2020 by the authors. Licensee MDPI, Basel, Switzerland. This article is an open access article distributed under the terms and conditions of the Creative Commons Attribution (CC BY) license (http://creativecommons.org/licenses/by/4.0/). 